\title{
P2P Mobile Multimedia Group Conferencing: Combining SIP, SSM and Scalable Adaptive Coding for Heterogeneous Networks ${ }^{\star}$
}

\author{
Thomas C. Schmidt ${ }^{1}$, Matthias Wählisch ${ }^{1,2}$, \\ Hans L. Cycon ${ }^{3}$, and Mark Palkow ${ }^{4}$ \\ ${ }^{1}$ HAW Hamburg, Dep. Informatik, Berliner Tor 7, D-20099 Hamburg, Germany \\ 2 link-lab, Hönower Str. 35, D-10318 Berlin, Germany \\ ${ }^{3}$ FHTW Berlin, FB I, Allee der Kosmonauten 20-22, D-10315 Berlin, Germany \\ ${ }^{4}$ daViKo GmbH, Am Borsigturm 40, D-13507 Berlin, Germany \\ \{t.schmidt, waehlisch\}@ieee.org \\ hcycon@fhtw-berlin.de, palkow@daviko.com
}

\begin{abstract}
In this paper we present work in progress on extending multimedia conferencing standards to scalable, mobile multimedia group support based on SIP initiated Source Specific IP Multicast. We propose extensions of SIP for negotiating SSM sessions. SIP protocol specifications and semantics are compatibly extended without adding new SIP methods. We will introduce a multimedia communication software with distributed architecture as implementation reference. The software is built on a scalable video codec adaptive to heterogeneous network capacities.
\end{abstract}

\section{Introduction}

Today's rapidly rising capabilities of devices and infrastructure, as well as an increasing social focus on ubiquitous infotainment will most likely establish mobile multimedia communication as a day-to-day companion. Real-time video conferencing and group communication thereby are likely to face acceptance not only as a particular, but a common service, raising questions of ease and quality of the underlying Internet service layer. Here IP multicasting will be of particular importance to mobile environments, where users commonly share frequency bands of limited and heterogeneous capacities.

In the present paper we address the issue of mobile multimedia group conferencing, exemplarily taking perspective on a VCoIP (Video Conferencing over IP) software with a distributed, peer-to-peer architecture and its applications [1. The software is build around a scalable video codec [2] which can serve heterogeneous network capacities. Such lightweight solution should receive support from network layer multicast, restricting service to Source Specific Multicast communication for the sake of deployment simplicity. Source Specific Multicast (SSM) [3], just released as an initial standard, is considered a promising improvement of group distribution techniques.

\footnotetext{
* Supported by the German BmBF within the Project Moviecast.
} 
However, up until now the session signalling standard SIP [4] is not prepared to negotiate SSM group sessions. We therefore present a straight forward extension of session initiation handshakes, suitable to establish SSM conferencing within an uncoordinated peer-to-peer model. Compliant with standard unicast and ASM transactions, we propose to solely add on protocol semantics without introducing new SIP methods. Our video conferencing system serves as a platform for reference implementation. We further reference to work on session mobility with the special focus on real-time multicast group communication. Conferencing parties request seamless real-time performance of a mobility aware group communication service, thereby attaining the simultaneous roles of mobile multicast listener and source. Up until now only limited work has been dedicated to multicast source mobility, which poses the more delicate problem [56].

In this paper we first briefly discuss the mobile multimedia group conferencing problem and related work. In section 3 we present our SIP signalling scheme for SSM group initiation. Section 4 is dedicated to a conclusion and an outlook.

\section{The Mobile Multimedia Group Conferencing Problem and Related Work}

Multimedia session-based communication requires a number of initial negotiations to be accomplished. At first, a caller requesting contact to one or several partners will expect to address a personal identifier, but establish the corresponding conference session with the devices currently in use by the callees. At second, media and service data need to be identified as to meet capabilities of all session members. Once established, sessions need to persist, while mobile devices roam. Intermediate handovers thereby should unnoticeably comply with quality of service measures for real-time communication.

SIP forms a multi-layered application protocol that interacts between components in a transactional way. Each (asynchronous) request initiates an open transaction state and requires completion by at least one response. Group communication complicates this process significantly. The basic SIP RFC only defines a minimal message exchange for Any Source IP layer multicast: A client wishing to initiate or join into a multiparty conference sends its INVITE request to a multicast group by employing the maddr attribute in the SIP VIA header. Group members subsequently respond to the same group. The transactional nature of SIP is preserved in the sense that the inviting party interprets the first arriving $\mathrm{OK}$ as the regular completion, while interpreting further messages as iterates. Consequently, SDP Negotiations on media parameters are displaced out-of-band, since they are not achievable within one transaction. While Any Source Multicast accounts for an open common addressing, SSM presupposes source specific subscriptions. Hence it requires a distribution of newly joining session member's addresses, which otherwise remain unnoticeable to the group.

Group conferencing must be considered a generic term for a wide variety of meanings in different contexts [7]. Three relevant types are essentially considered: In loosely coupled conferences, no signalling relationship is maintained 
by conference parties, while meta information are pre-shared out of band or gradually learned by RTCP control streams. Tightly coupled conferences rely on central managing for all participants. Finally, the fully distributed, infrastructureless multiparty model is built upon peer-to-peer signalling. Its scalability can be largely enhanced by the use of multicast. Further on we will concentrate on this fully distributed approach, including SSM at the signalling level.

Without optional proxy servers the SIP protocol architecture draws near the general peer-to-peer model. Recent IETF work aims on extending the SIP framework to include fully distributed infrastructureless approaches [8]. Additional work is needed to develop peer-to-peer group support within a SIP control plane. Keeping in mind the routing complexity inherent to ASM, it is desirable to rigorously restrict all signalling to unicast or SSM communication.

\section{SIP Initiated SSM Group Conferences}

Instantaneous establishment of a fully distributed peer-to-peer conference commonly follows an incremental setup: Some party will initiate a conference by contacting one or several peers via unicast. Following an initial contact, signalling will then be turned to scalable multicast. Further on new parties will join the conference by either calling or being called by an existing member. Such group initiation scheme is neither covered by the current status of SIP, nor is the employment of Source Specific Multicast for signalling. In order to enable SSM, all dialogs must carefully provision addresses of newly arriving senders to all current group members to allow for appropriate source specific subscriptions.

In detail protocol operations of the suggested extensions proceed as follows. A caller, wishing to establish an SSM signalling session with a single peer, will initiate a regular INVITE request to callee's unicast address. Eventually, after the call setup has completed, either party will decide to turn the established session to group communication. Heading for SSM, it will submit a re-INVITE, i.e., an INVITE carrying the previously established session identifier, announcing its desired multicast group address in the CONTACT field of the SIP header. Concomitantly, the SIP protocol stack will submit a multicast source specific JOIN to its underlying IGMP/MLD stack for subscribing to the group and peer's source address, which it had both learned from the previous SIP message exchange. Any peer will identify the multicast address in the CONTACT field, designed to specify contacts for subsequent requests, and proceed along the new protocol semantic for SSM. As ASM multicast address announcement will distinctively appear in a separate VIA header, it will identify the presence of SSM, answer with a regular unicast reply, but will submit a multicast JOIN to the announced group and caller's source address. This two-step process purposefully decouples application layer session establishment and underlying multicast routing operations. Temporal progress in IP-layer multicast routing and SIP transactional timers thereby remain independent for the sake of robustly layered protocol operations. Appropriate media session descriptions for SSM distribution of media streams may be negotiated along the re-INVITE request. 
In multiparty environments, a newly arriving party will contact some member of the established session via regular unicast INVITE. The callee might decide to accept this request and forward it to its partner, thereby initiating unicast sessions among the three. Eventually the callee will decide to select multicast for the conference signalling and will submit the corresponding re-INVITE procedure.

If a new source $S_{\text {new }}$ contacts an established SSM group conference, it will do so by inviting some member $S$. If $S$ decides to accept the caller, it will redistribute its INVITE to the SSM group and acknowledge the initial call by placing the group address in the CONTACT header field. All group members will immediately add $S_{n e w}$ to their source specific multicast filters. $S_{n e w}$ subsequently will learn about all group members from (unicast) OK-messages as needed for its own multicast subscriptions. Note that call redistribution will remain a pointto-point request of $S_{\text {new }}$ at the application layer, but a transmission of source $S$ at the network layer compliant with previous SSM group establishment.

\section{Conclusions and Outlook}

In this paper we addressed the essential issues of multimedia group conferences, negotiated by the Session Initiation Protocol and relying on Source Specific Multicast distribution among equal peers. We introduced peer-to-peer signalling extensions of SIP. Proceeding along the proposed incremental way, a callee will never be required to communicate messages to more than one party and one group. This scheme thus remains fully scalable and fairly transparent to group sizes. Multicast initiation of media sessions may be led correspondingly. We also reported on a peer-to-peer reference multimedia communication software with temporal scalable video codec as core module and referred to routing methods to adapt SSM to source mobility. This enables conferencing in networks with heterogeneous capabilities. Implementations for real deployment are under way.

\section{References}

1. Palkow, M.: The daViKo homepage (2006) http://www.daviko.com.

2. Schwarz, H., Marpe, D., Wiegand, T.: Overview of the Scalable H.264/MPEG4AVC Extension. In: IEEE International Conference on Image Processing (ICIP 2006), Atlanta, GA, USA (2006)

3. Holbrook, H., Cain, B.: Source-Specific Multicast for IP. RFC 4607, IETF (2006)

4. Rosenberg, J., Schulzrinne, H., Camarillo, G., Johnston, A., Peterson, J., Sparks, R., Handley, M., Schooler, E.: SIP: Session Initiation Protocol. RFC 3261, (2002)

5. Schmidt, T.C., Wählisch, M.: Multicast Mobility in MIPv6: Problem Statement. IRTF Internet Draft - work in progress 01, MobOpts (2006)

6. Schmidt, T.C., Wählisch, M.: Morphing Distribution Trees - On the Evolution of Multicast States under Mobility and an Adaptive Routing Scheme for Mobile SSM Sources. Telecommunication Systems 33 (2006) 131-154

7. Rosenberg, J.: A Framework for Conferencing with the Session Initiation Protocol (SIP). RFC 4353, IETF (2006)

8. Baset, S., Schulzrinne, H., Shim, E., Dhara, K.: Requirements for SIP-based Peerto-Peer Internet Telephony. Internet Draft - work in progress 00, IETF (2006) 